\title{
Left-right facial orientation of familiar faces: developmental aspects of " the mere exposure hypothesis "
}

\author{
Anouck Amestoy ${ }^{1,2}$, Manuel P. Bouvard ${ }^{1,2}$ and Jean-René Cazalets ${ }^{2 *}$ \\ Child Psychiatry Department, Université de Bordeaux, Bordeaux, France \\ 2 Centre National de la Recherche Scientifique, Université de Bordeaux, Bordeaux, France
}

Edited by:

Rufin VanRullen, Centre de Recherche Cerveau et Cognition, France

\section{Reviewed by:}

Bruno Rossion, Université Catholique de Louvain, Belgium

Cathy Mondloch, Brock University,

Canada

Danna Kuefner, Universite Catholique

de Louvain, Belgium

*Correspondence:

Jean-René Cazalets, Centre National

de la Recherche Scientifique UMR

5227, Université Bordeaux 2, Zone nord

Bât 2, 2e étage, 146, rue Léo Saignat,

33076 Bordeaux Cedex, France.

e-mail: jean-rene.

cazalets@u-bordeaux2.fr
We investigated the developmental aspect of sensitivity to the orientation of familiar faces by asking 38 adults and 72 children from 3 to 12 years old to make a preference choice between standard and mirror images of themselves and of familiar faces, presented side-by-side or successively. When familiar (parental) faces were presented simultaneously, 3- to 5-year-olds showed no preference, but by age 5-7 years an adult-like preference for the standard image emerged. Similarly, the adult-like preference for the mirror image of their own face emerged by $5-7$ years of age. When familiar or self faces were presented successively, 3- to 7-yearolds showed no preference, and adult-like preference for the standard image emerged by age 7-12 years. These results suggest the occurrence of a developmental process in the perception of familiar face asymmetries which is retained in memory related to knowledge about faces.

Keywords: facial asymmetries, mirror image, familiar faces, development, implicit memory

\section{INTRODUCTION}

Faces are the most complex and frequently encountered social visual stimulus that has to be not only processed preferentially and rapidly compared to other categories of visual stimuli (Crouzet et al., 2010), but also accurately to ensure recognition. Human faces, however, are not symmetrical, and various studies have demonstrated a sensitivity to minor facial details and left-right asymmetries around the central axis which affects the recognition process related to facial likeness, identity, gender, attractiveness, and expression in adults. Such perceptual asymmetries in face information processing have to date been mostly reported with stimuli employing chimeric faces and the results obtained indicated a robust left perceptual effect (Burt and Perrett, 1997; Chen et al., 1997; Quinn et al., 2008). It was assumed that these effects may be due to a right hemisphere bias in the face recognition processing, or, according to other authors, derive from an interaction between a habitual directional eye scanning bias and a cerebral lateralization (Ferber and Murray, 2005; Butler and Harvey, 2006).

Several studies, referring to the "mere exposure " hypothesis (repeated exposure of the individual to a stimulus object enhances his attitude toward it; Zajonc, 1968), have explored the role of experience in face processing, and demonstrated that adult facial memory includes a representation of left-right facial orientation. In pioneering experiments that investigated the representation of left-right facial orientation in adults, participants showed a significant preference for the mirror image of their own faces, while their friends preferred the unreflected standard images (Mita et al., 1977). Here, the stimulus that was seen more frequently received a more favorable evaluation in accordance with the mere exposure hypothesis, which supports the idea that subjects develop stronger preferences for objects that have become familiar through repeated exposure. Several lines of evidence suggest that the mere exposure effect is based on implicit memory (Seamon et al., 1995; Willems et al., 2002), even when preference choice is requested (Willems et al., 2002). This effect has proven to be a robust, reliable phenomenon and has been demonstrated using a variety of stimuli (for review, see Harrison, 1977; Bornestein, 1989). Studies including amnesic and prosopagnosic patients offer arguments for postulating that the mere exposure effect is an implicit memory manifestation (e.g., Bremner et al., 2000). Here we have tested this effect in order to explore the sensitivity and memory of facial orientation during childhood. More recently, similar results were found in adults performing a discriminative recognition task, but not a preference task, which suggests that memory for familiar faces incorporates canonical or common views and a representation of facial configuration (Rhodes, 1986; Bredart, 2003; Brady et al., 2004, 2005).

In addition to these findings in adults, developmental studies have suggested that face processing involves an innate system that guides attention to faces (Fantz, 1963; Johnson et al., 1991), and develops proficiency early and quickly during childhood, thus suggesting an interaction between nervous system maturation and experience (Blaney and Winograd, 1978; Diamond and Carey, 1986; Ellis and Flin, 1990; Ge et al., 2008). In this way, face processing is thought to reflect a developmental progression in the encoding strategy, from the use of featural to configural (relational) information, partly as a consequence of experience. Particularly, this transition is thought to underlie the improvement in facial recognition performance from 5 - to 12 -year-old children and the question is when this sensitivity becomes adult-like (Carey et al., 1980; Mondloch et al., 2006; Pascalis and Kelly, 2009). 
New recent findings have supported the attractive idea that adult-like features of face processing are present much earlier in development, by the age of 4 years at the latest (McKone et al., 2009). Furthermore, the role of face experience in the developmental course of face recognition has been reinterpreted. On the basis of an innate system that provides a representational capacity for differentiating individual face structure, experience may then play a role in narrowing the range of facial subtypes for which discrimination is possible and perhaps also in increasing discriminatory capabilities within the experienced range (for review see McKone et al., 2009).

Any developmental changes in sensitivity to left-right orientation may result from a precocious perceptual and memory capability. In the present study, therefore we investigated whether the perception and memory of facial asymmetries in the vertical axis is present early in development and if it follows the chronology of general facial recognition. In the context of the mere hypothesis, preference choice allowed us to compare response differences that were so slight that they were just barely accessible to the subject's consciousness (Mita et al., 1977). This allowed us to explore the familiar aspect of the stimuli in children (Kunst-Wilson and Zajonc, 1980; Bornstein, 1989), and the notion that children become progressively more sensitive to minimal face asymmetry between 3 and 12 years of age.

\section{MATERIALS AND METHODS}

One hundred and ten individuals participated in the experiment including, 38 adults (28-60 years, mean 42.3; 18 females and 20 males) and 72 children (3-12 years separated in three groups: 24 children from 3 to 5 years, mean $4.1 ; 24$ children from 5 to 7 years, mean $6.0 ; 24$ children from 7 to 12 years, mean $10.3 ; 40$ females and 32 males). The age groups were defined a posteriori, based on the emergence of a significant preference choice. Parental permission was obtained for each child, and in accordance with ethical guidelines, parents were assured that photographs would be used solely for the purpose of the experiment. The adult sample consisted of researchers in the same neuroscience department as the authors. Participants were paired with colleagues who worked in the same office for at least 1 year while children were recruited from a local leisure community center. The familiar face presented to the children was a parent's face.

Photographs were taken face-on with a neutral expression under symmetrical lighting and in front of a plain background. Each subject had to examine 4 dyads: standard and mirror images of self and of a familiar face presented successively and side-by-side. Each slide was presented for $4 \mathrm{~s}$ and separated by a black screen for $4 \mathrm{~s}$. The position of the standard and mirror images as well as the order of presentation condition, i.e., side-by-side and sequentially, were randomized. We used dual presentation, since it was possible that the side-by-side presentation alone, could induce a comparative effect that would immediately reveal the mirror symmetry between the two views. Participants were requested at the end of each dyad to make a preference choice on first impression. We restricted our protocol to a 4-dyad presentation to avoid the possibility that multiple repetitive presentations of several faces progressively evoked questioning about the nature of the differences between the slides, which in turn would have progressively biased the subject's responses.
Our standardized protocol limited such a bias. Furthermore, in order to compare our developmental data (and those replicated in adult controls) to previous studies performed solely on adults (Mita et al., 1977), we employed a comparable protocol to test face presentation, i.e., with all facial attributes retained and not with cropped photos at the hairline.

In order to assess whether the children, and particularly the youngest subjects (3-5 years) truly understood the concept of the preference task, we designed a training task (Cooper et al., 2006), composed of a series of dyads that included various items (for example, lollipop vs candy or gray fluff vs brown fluff...). Half of the 3-5-year-old child sample (12 out of 24) was tested in this task.

To ensure that differences were not due to specific features of the images themselves (symmetry of hairstyles or clothes, light...) a comparable paradigm was applied to a separate sample of 25 adults who were unfamiliar with the face presented. They were asked to view a series of 20 dyads that were randomly selected from those presented to the control adult groups.

Statistical analyses (SPSS, Chicago, IL, USA) was performed using tests of significance of binomial proportion.

\section{RESULTS}

In the adult group, when pictures were presented side-by-side, $71 \%$ (27 of 38 ) preferred their own mirror image, a result that was significantly different from chance $[p=0.014$, binomial $(27$, $38,1 / 2)$ ]. Similarly, the proportion of adults who preferred the standard image of their friend $(76 \%, 29$ of 38$)$ was statistically significant $[p<0.01$, binomial $(29,38,1 / 2)]$.

When pictures were presented successively, $71 \%$ of adult participants chose their own mirror image $[p=0.014$, binomial $(27,38$, $1 / 2)]$ while $84 \%$ preferred their friend's standard image $[p<0.001$, binomial $(32,38,1 / 2)]$. The difference between the proportion of positive answers for « self » preference and « familiar » preference reached significance [32 and 27 respectively, Mc Nemar's test, chisquare $=12.1$, with 1 degree of freedom $(p<0.001)$ ], suggesting that memory of a friend's face was stronger than self face memory in this condition.

Results from the three different child groups suggested a developmental progression in the differences between «familiar » face and « self » face preference (Figure 1).

When performing the training task (see Materials and Methods), all the 3- to 5-year-olds tested, immediately understood the task and had no difficulty in performing the preference choice. Indeed this was also the case for older subjects.

In the side-by- side condition, $46 \%$ of the 3 - to 5 -year-olds preferred the mirror image of themselves $[p=0.839$, binomial $(13,24,1 / 2)]$, and $62 \%$ preferred their parent's standard image $[p=0.3$, binomial $(15,24,1 / 2)]$. In contrast, the results from the 5 to 7 years old group matched those from the adult sample. A significant $79 \%$ (19 of 24) of 5- to 7-year-old children preferred their parents' standard image $[p=0.07$, binomial $(19,24,1 / 2)]$, and $75 \%$ (18 of 24$)$ preferred their own mirror image $[p=0.023$, binomial $(18,24,1 / 2)]$. In the third 7 - to 12 -year-old group, a significant $83 \%$ (20 of 24) preferred the standard image of their parents while $75 \%$ preferred their own mirror image $[p=0.023$, binomial $(18,24,1 / 2)]$. 


\section{SIDE BY SIDE}

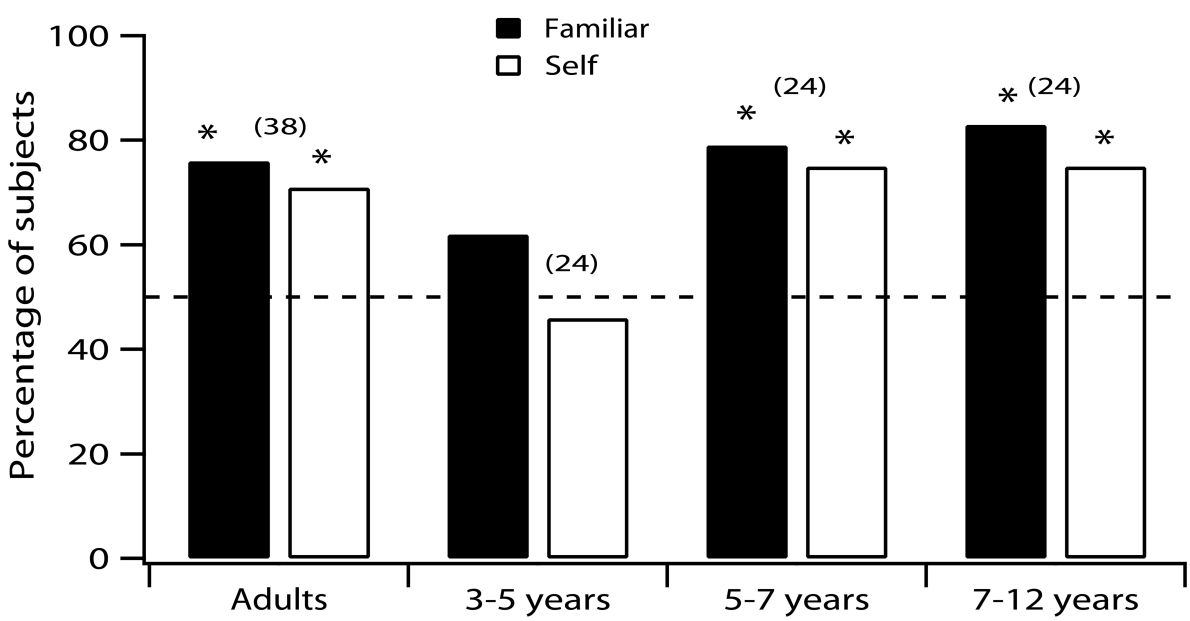

SUCCESSIVE

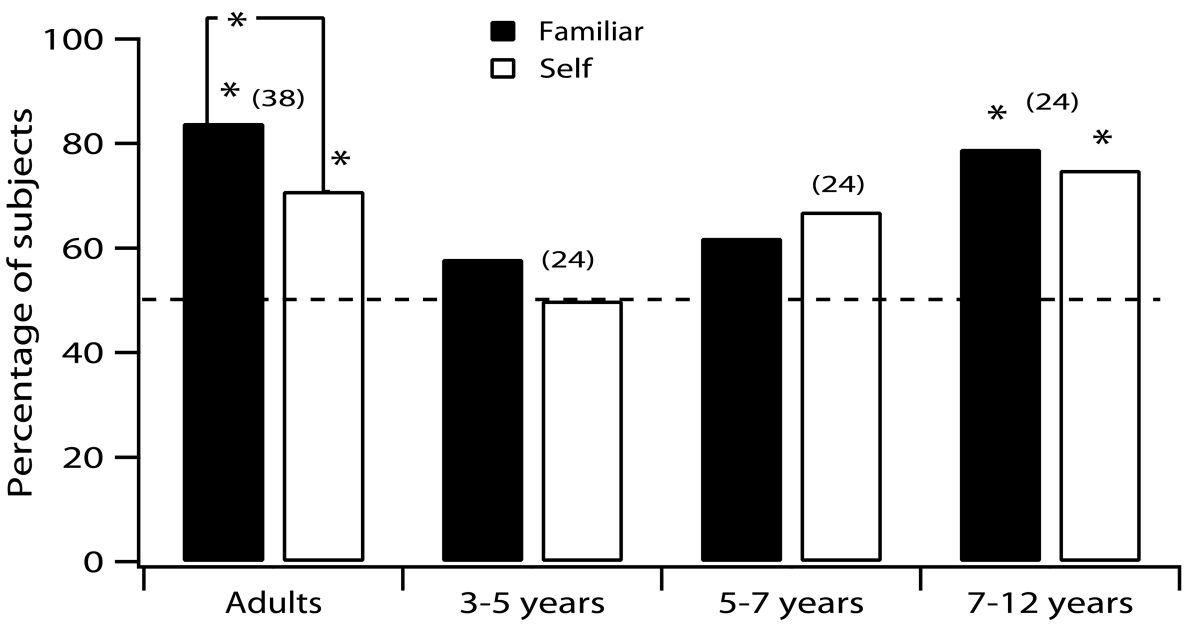

FIGURE 1 | Changes in the preference choice through development and in adults. The horizontal dashed line (at $50 \%$ ) indicates chance.

In the successive condition, $50 \%$ of 3 - to 5 -year-olds preferred the standard self image $[p=1$, binomial $(12,24,1 / 2)]$, and $62 \%$ preferred their parent's standard image $[p=0.3$, binomial $(15,24,1 / 2)]$.

Similarly, $67 \%$ of the 5 - to 7 -year-olds preferred the mirror image of themselves $[p=0.152$, binomial $(16,24,1 / 2)]$, and $62 \%$ preferred their parent's standard image $[p=0.3$, binomial (15, $24,1 / 2)]$. Preference choice appeared to differ significantly from chance only in the eldest group (7-12 years), for both dyads of self and familiar faces that were chosen respectively by 18 and 19 of 24 children $[p=0.023$, binomial $(18,24,1 / 2)$, and $(p=0.007$, binomial $(19,24,1 / 2)]$.

In the side-by-side presentation condition, we found no significant differences between participant choices of right or left image. Similarly, in the successive condition, we found no significant differences between participant choices of first or second image.
When participants were asked to indicate the basis for their selection, they mentioned " better facial angle ", " modified photographs ", « better head tilt », " looks prettier, older »... They sometimes evoked unfounded differences, in thinking that pictures were modified (e.g., added wrinkles). When the purpose of the study was described, participants mentioned the exploration of differences in perception of minor face variation. Only six adults suggested symmetries or mirror-inversion effects. Reasons of choice were more difficult to explore with children. Some of them mentioned « hair style », « smiling or frowning », but none of them had thought of a mirror view. Data for all trials (successively and side-by-side), and groups are presented in Figure 1. The 25 control adults who were unfamiliar with the presented faces (see Materials and Methods) showed no significant differences in their preference choices between the standard or mirror images of the same face. 


\section{DISCUSSION}

This study investigated whether the perception of asymmetries of highly familiar faces, already documented in adults (Mita et al., 1977), is present early in child development. We found that sensitivity to minor facial details such as face orientation around the vertical axis could enhance preference after 5 years of age. The traditional preference task used in our experiment exploits the visual system's sensitivity to subconscious and conscious details in a discriminatory task involving familiarity. Novel to our study is the finding of an early and accurate development of the perception and memory of facial asymmetries, in accordance with developmental data documented in general face processing.

Early developmental studies have already suggested that face recognition in childhood undergoes a learning and biological maturational process (Blaney and Winograd, 1978; Carey et al., 1980; Ellis and Flin, 1990; Chung and Thomson, 1995). More recent behavioral research has suggested that a standard adult face recognition capability is present in young children with many properties investigated, including discriminatory effects on perception and memory having been observed in 4 year and 5- to 6-year-olds respectively (Gilchrist and McKone, 2003; McKone and Boyer, 2006; McKone et al., 2009). Our results are in accordance with this general framework of the developmental trajectory of face perception and memory, suggesting an access to an acute representation of a very familiar face, at least after 5 years of age, then reaching full adult competence between the ages of 7 and 12 years.

This last development in face processing performances may be due to general factors as suggested by differences between results in the side-by-side condition and the successive condition in the 5- to 7-year-old group, which disappear in the eldest group of 7- to 12-year-old group, consistent with general cognitive development theory (Crookes and Mc Kone, 2009). In fact, 5- to 7-year-old children did not choose the standard orientation of their parents' face or the mirror orientation of themselves when images were presented successively, although they exhibited face orientation sensitivity in the side-by-side condition. This may reflect a contribution of the cognitive charge, which is stronger in the first condition which is likely to involve a combination of acute working memory, attention, and perception. This finding thus adds new support for the hypothesis of early performance in face recognition and memory, which is tightly linked to the development of other cognitive functions involved in the process.

\section{REFERENCES}

Blaney, R. L., and Winograd, E. (1978). Developmental differences in children recognition memory for faces. Dev. Psychol. 14, 441-442.

Bornstein, R. F. (1989). Exposure and affect: overview and meta-analysis of research, 1968-1987. Psychol. Bull. 106, 265-289.

Brady, N., Campbell, M., and Flaherty, M. (2004). My left brain and me: a dissociation in the perception of self and others. Neuropsychologia 42, 1156-1161.

Brady, N., Campbell, M., and Flaherty, M. (2005). Perceptual asymmetries are preserved in memory for highly

Our results from adults are in agreement with previous studies, showing that face orientation is retained in memory for familiar faces (own face and friend's face), and that this memory influences judgment even if adults were not required to answer as to which of the two images looked like themselves or their friends. Furthermore, the same result was obtained whether the subject was presented with dyads in side-by-side or successive modes. Surprisingly, our data also suggest that an adult's memory of the orientation of a friend's face is stronger than the corresponding memory of his/her own face in both presentation conditions but particularly if faces are shown successively. It has been suggested that people have the same ability to recognize the correct orientation of their own or another's face, but with a prominent role of configural information in a friend's face recognition whereas asymmetrically located details seems to be more relevant in our own face choice (Bredart, 2003).

That our results from adults are in agreement with other previous studies (Mita et al., 1977; Rhodes, 1986; Brady et al., 2004) also validates the protocol used here. Furthermore, our study shows for the first time that facial orientation seems to be such a salient feature in memory that even if a direct comparison between the two facial orientations is not possible (due to successive image presentation), the standard view affects judgment in the expected way. This is particularly evident in the " friend " condition, since the proportion of expected answers is the highest. One hypothesis is that comparative processing in the side-by-side condition is likely to involve a screening strategy in which an active search for differences may affect spontaneous answers via more implicit processes.

Beside the issue of the role of perception and expertise in the development of highly familiar face memory, the degree of salience of the hemispheric asymmetries (Brady et al., 2005) and the emotional salience when studying self face and familiar face processing remain major issues that also require investigation in the context of childhood maturation and development (Richards and Gross, 2000).

\section{ACKNOWLEDGMENTS}

The authors thanks L. Demany and O. Pascalis for their valuable comments on the manuscript and N. Mellen and J. Simmers for correcting the English. Many thanks to C. Goulet for kind advices on statistical analyses. This work was funded in part by the « Région Aquitaine».

Butler,S.H.,andHarvey,M.(2006).Perceptual biases in chimeric face processing: eyemovement patterns cannot explain it all. Brain Res. 1124, 96-99.

Carey, S., Diamond, R., and Woods, B. (1980). Development of face recognition-a maturational component? Dev. Psychol. 16, 257-269.

Chen, A. C., German, C., and Zaidel, D. W. (1997). Brain asymmetry and facial attractiveness: facial beauty is not simply in the eye of the beholder. Neuropsychologia 35, 471-476.

Chung, M.S., and Thomson, D.M.(1995). Development of face recognition. $\mathrm{Br}$. J. Psychol. 86, 55-87.
Cooper,P.A., Geldart, S.S., Mondloch, C.J., and Maurer, D. (2006). Developmental changes in perceptions of attractiveness: a role of experience? Dev. Sci. 9 , 530-543.

Crookes, K., and McKone, E. (2009). Early maturity of face recognition: no childhood development of holistic processing, novel face encoding, or face-space. Cognition 111, 219-247.

Crouzet, S. M., Kirchner, H., and Thorpe, S. J. (2010). Fast saccades toward faces: face detection in just $100 \mathrm{~ms}$. J. Vis. $10,1-17$.

Diamond, R., and Carey, S. (1986). Why faces are and are not special - an 
effect of expertise. J. Exp. Psychol. 115, 107-117.

Ellis, H. D., and Flin, R. H. (1990). Encoding and storage effects in 7-yearolds' and 10-year-olds' memory for faces. Br. J. Dev. Psychol. 8, 77-92.

Fantz, R. L. (1963). Pattern vision in newborn infants. Science 140, 296-297.

Ferber, S., and Murray, L. J. (2005). Are perceptual judgments dissociated from motor processes? - A prism adaptation study. Brain Res. Cogn. Brain Res. 23, 453-456.

Ge, L., Anzures, G., Wang, Z., Kelly, D. J., Pascalis, O., Quinn, P. C., Slater, A. M., Yang, Z., and Lee, K. (2008). An inner face advantage in children's recognition of familiar peers. J. Exp. Child. Psychol. 101, 124-136.

Gilchrist, A., and McKone, E. (2003). Early maturity of face processing in children: local and relational distinctiveness effects in 7-year-olds. Vis. Cogn. 10, 769-793.

Harrison, A. A. (1977). "Mere exposure," in Advances in Experimental Social Psychology, ed. L. Berkowitz (New York: Academic Press), 39-83.

Johnson, M. H., Dziurawiec, S., Ellis, H., and Morton, J. (1991). Newborns' preferential tracking of face-like stimuli and its subsequent decline. Cognition 40, 1-19.

Kunst-Wilson, W. R., and Zajonc, R. B. (1980) Affective discrimination of stimuli that cannot be recognized. Science 207, 557-558.

McKone, E., and Boyer, B. L. (2006). Sensitivity of 4-year-olds to featural and second-order relational changes in face distinctiveness. J. Exp. Child. Psychol. 94, 134-162.

McKone, E., Crookes, K., and Kanwisher, N. (2009). The cognitive and neural development of face recognition in humans. in The Cognitive Neurosciences. ed. S. Gazzaniga (Cambridge, MA: MIT Press), 467-482.

Mita, T. H., Dermer, M., and Knight, J. (1977). Reversed facial images and the mere-exposure hypothesis. J. Pers. Soc. Psychol. 35, 597-601.

Mondloch, C. J., Maurer, D., and Ahola, S. (2006). Becoming a face expert. Psychol. Sci. 17, 930-934.

Pascalis, O., and Kelly, D. J. (2009). The origins of face processing in humans: phylogeny and ontogeny. Perspect. Psychol. Sci. 4, 200-209.
Quinn, P. C., Kelly, D. J., Lee, K., Pascalis, O., and Slater,A.M. (2008). Preference for attractive faces in human infants extends beyond conspecifics. Dev. Sci. $11,76-83$.

Rhodes, G. (1986). Memory for lateral asymmetries in well-known faces: evidence for configural information in memory representations of faces. Mem. Cogn. 14, 209-219.

Richards, J. M., and Gross, J. J. (2000). Emotion regulation and memory: the cognitive costs of keeping one's cool. J. Pers. Soc. Psychol. 79, 410-424.

Seamon, J., Williams, P., Crowley, M., Kim, I., Langer, S., Orne, P., and Wishengrad, D. (1995). The mere exposure effect is based on implicit memory - effects of stimulus type, encoding conditions, and number of exposures on recognition and affect judgments. J. Exp. Psychol. Learn. Mem. Cogn. 21, 711-721.

Willems, S., Adam, S., and Van der Linden, M. (2002). Normal mere exposure effect with impaired recognition in Alzheimer's disease. Cortex 38 77-86.
Zajonc, R. B. (1968). Attitudinal effects of mere exposure. J. Pers. Soc. Psychol. 9, 1-8.

Conflict of Interest Statement: The authors declare that the research was conducted in the absence of any commercial or financial relationships that could be construed as a potential conflict of interest.

Received: 15 January 2010; paper pending published: 27 March 2010; accepted: 13 July 2010; published online: 14 September 2010. Citation:AmestoyA,BouvardMPand Cazalets $J-R$ (2010) Left-right facial orientation of familiar faces: developmental aspects of « the mere exposure hypothesis». Front. Psychology 1:39. doi: 10.3389/fpsyg.2010.00039

This article was submitted to Frontiers in Perception Science, a specialty of Frontiers in Psychology.

Copyright (๑) 2010 Amestoy, Bouvard and Cazalets. This is an open-access article subject to an exclusive license agreement between the authors and the Frontiers Research Foundation, which permits unrestricted use, distribution, and reproduction in any medium, provided the original authors and source are credited. 